\title{
Macrobend optical sensing for pose measurement in soft robot arms
}

Sina Sareh ${ }^{\mathrm{a}}$, Yohan Noh ${ }^{\mathrm{a}}$, Min Li ${ }^{\mathrm{b}}$, Tommaso Ranzani ${ }^{\mathrm{c}}$, Hongbin Liu ${ }^{\mathrm{a}}$, Kaspar Althoefer ${ }^{\mathrm{a}}$

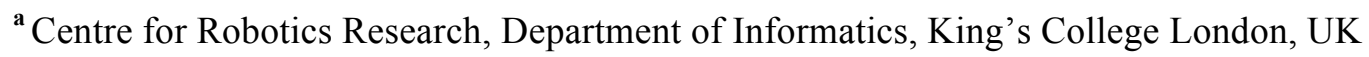

${ }^{\mathrm{b}}$ Institute of Intelligent Measurement \& Instrument, School of Mechanical Engineering, Xi'an Jiaotong University, China

${ }^{\mathrm{c}}$ Harvard John A. Paulson School of Engineering and Applied Sciences, Wyss Institute for Biologically Inspired Engineering, Harvard University, Cambridge, MA, USA

Email: $\underline{\text { Sina.Sareh@,kcl.ac.uk }}$

This paper introduces a pose sensing system for soft robot arms integrating a set of macrobend stretch sensors. The macrobend sensory design in this study consists of optical fibres and is based on the notion that bending an optical fibre modulates the intensity of the light transmitted through the fibre.

This sensing method is capable of measuring bending, elongation and compression in soft continuum robots and is also applicable to wearable sensing technologies, e.g. pose sensing in the wrist joint of a human hand. In our arrangement, applied to a cylindrical soft robot arm, the optical fibres for macrobend sensing originate from the base, extend to the tip of the arm, and then loop back to the base. The connectors that link the fibres to the necessary opto-electronics are all placed at the base of the arm, resulting in a simplified overall design.

The ability of this custom macrobend stretch sensor to flexibly adapt its configuration allows preserving the inherent softness and compliance of the robot which it is installed on. The macrobend sensing system is immune to electrical noise and magnetic fields, is safe (because no electricity is needed at the sensing site), and is suitable for modular implementation in multi-link soft continuum robotic arms.

The measurable light outputs of the proposed stretch sensor vary due to bend-induced light attenuation (macrobend loss), which is a function of the fibre bend radius as well as the number of repeated turns. The experimental study conducted as part of this research revealed that the chosen bend radius has a far greater impact on the measured light intensity values than the number of turns (if greater than five). Taking into account that the bend radius is the only significantly influencing design parameter, the macrobend stretch sensors were developed to create a practical solution to the pose sensing in soft continuum robot arms. Henceforward, the proposed sensing design was benchmarked against an electromagnetic tracking system (NDI Aurora) for validation.

\section{Introduction}

Soft continuum robots are typically made from soft materials that change their shape as dictated by the actuation system as well as to surfaces with which they are interacting (Trivedi et al 2008; Stilli et al 2014; Sareh et al 2012; Pfeifer and Iida 2009; Culha et al 2014). However, this ability of soft robots to morph their shape comes at the expense of complicating configuration and position control (Marchese et al 2014). 
To implement a pose control system for this class of robots, the deformation of the soft body must be monitored and incorporated into the control algorithm during the operation. A number of researches employ visual 3D tracking systems, e.g. the Vicon ${ }^{\circledR}$ Motion Capture system (MoCap) to collect information on the geometry of soft robots (Dobrzynski et al 2011; Saunders et al 2011) and continuum robots (Hennan et al 2003; Webster et al 2006). However, exploiting vision-based sensing systems requires a workspace sparsely populated with obstacles and sufficient space for installation of the sensor system. Electromagnetic tracking systems, e.g. NDI Aurora (Northern Digital Inc., Canada), can sense the position and orientation of finite numbers of magnetic tracking coils embedded inside or attached to the robot (Xu et al 2013). However, these systems are subject to magnetic field distortions (Kim et al 2014) and have limitations with regards to the mobility of the magnetic field generation system.

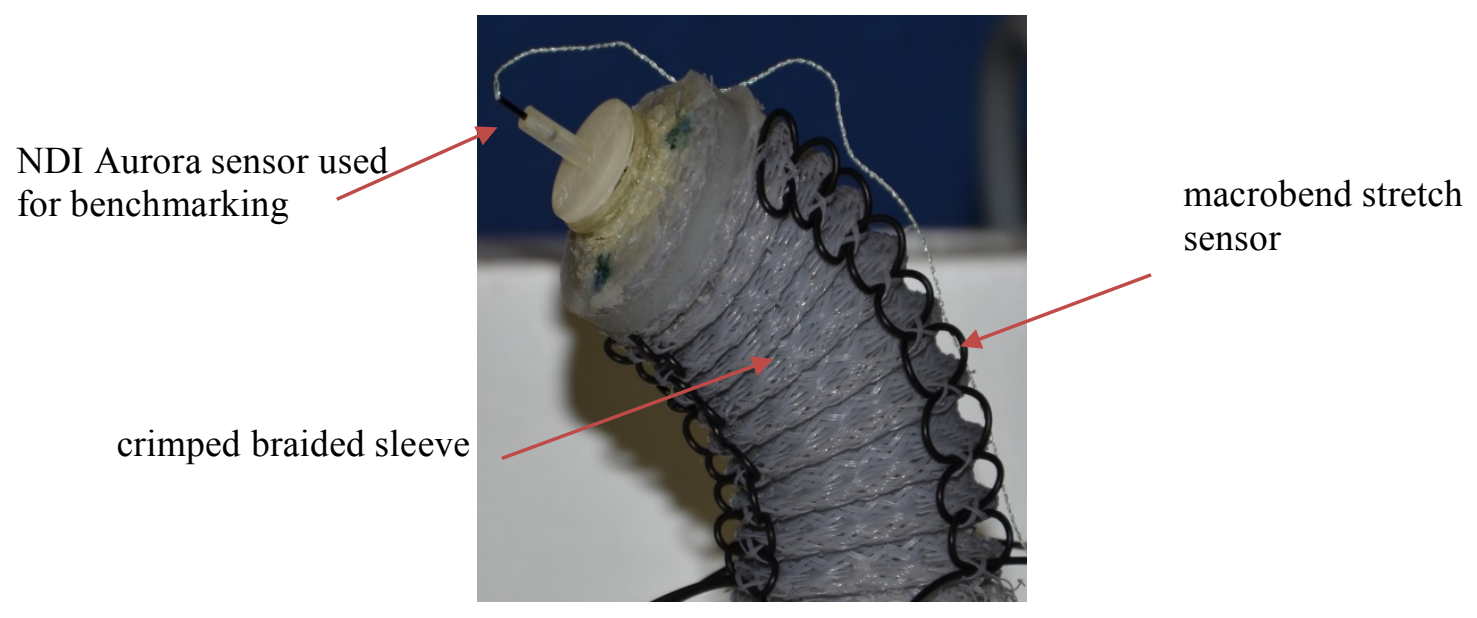

Figure 1. The side view of the sensorized soft arm. The pose sensing system consists of three custom macrobend stretch sensors sewn along the periphery of the arm which are equally orientated from one another at $120^{\circ}$ around the central axis of the arm.

A practical solution to achieve 3D shape sensing is to create an assembly of (at least) three length sensors arranged in parallel, as illustrated in Figure 2(b). When the assembly undergoes a specific amount of bending in a particular orientation, a unique combination of three lengths is measured by the sensors which can describe the configuration parameters of the assembly including the elongation, bending angle and orientation. These shape sensing systems employ various types of length sensors based on cable displacement, stretch and strain measurements.

Shape sensing systems based on passive cable displacement (Murphy et al 2014; Rone and Ben-Tzvi 2013) are influenced by the cables' sliding friction (Kim et al 2014), and possible stretch of the cables. Also, as passive cables of the distal segments have to be passed through the proximal segments of the arm, these sensing systems are usually impractical for modular implementations (Camarillo et al 2008).

In another approach, stretch sensors were integrated into the body of soft robots to sense the bending and elongation (Culha et al 2014; Cianchetti et al 2012). One of the best examples of off-the-shelf stretch sensors is based on conductive rubber (Images Scientific Instruments Inc, USA). Although, the conductive rubber sensors can be used for measuring the length via simple resistive circuits, they suffer from large relaxation times and hysteresis (Vibhute and Kshirsaga 2012). Another type of stretch sensors are based on dielectric elastomers and are supplied by StretchSense Ltd (New Zealand) and Danfoss PolyPower A/S 
(Denmark). These sensors are able to accurately measure the stretched length by a near linear voltage-length relationship. However, decoupling between different modes of sensing, e.g. pressure and stretch modes, is complex (O’Brien et al 2014).

Optical Fibre technology offers advantageous sensing capabilities for transducing strain, temperature, and force. The optical fibre sensors are immune to the magnetic field and are inherently safe for medical and industrial applications (Polygerinos et al 2010). Optical fibre sensing is based on the modulation of the optical characteristics of the light transmitted through the optical fibre, including light intensity (Liu et al 2010; Liu et al 2011; Sareh et al 2014; Noh et al 2014; Noh et al 2015), wavelength (Patrick et al 1998; Allsop et al 2005), and polarization (Feng et al 2011). Light intensity modulation has also been employed for pose sensing in soft robotics, by using two separate segments of optical fibres, one connected to the emitter and the other one to the detector (Sareh et al 2015a; Sareh et al 2015b; Searle et al 2013). Fibre Bragg Grating (FBG) sensors have been used for shape sensing in continuum robots (Roesthuis et al 2013). However, FBG systems are highly sensitive to strain conditions. When subjected to a non-uniform strain field, the strain compensation becomes complex (Zhang et al 2007).

Bending an optical fibre is another approach to modulate the intensity of the light transmitted via the fibre. Macrobend optical sensing can be realized through sufficient bending of the optical fibre beyond the point at which the critical angle for light reflection is exceeded (Jay 2010). The macrobending of an optical fibre connected to a light source leads to light loss along the length of the fibre that can be measured using a light detector, e.g. FS-N11MN fibre optic sensor (Keyence ${ }^{\mathrm{TM}}$, USA). This desired light loss can occur at multiple locations along the length of the fibre (Silva et al 2013). In Zawawi et al 2013, implementations of macrobend stretch sensors were proposed in semicircular (half turn), circular (one turn), and figure-of-eight configurations. Other prominent examples include sinusoidal and roller coaster loop-the-loops configurations (Silva et al 2013). Macrobend sensing method has also been employed in a multi-layer configuration for plantar pressure and shear sensing (Wang et al 2005), for the development of a temperature sensor (Moraleda et al 2013), and for a voltage sensor (Wang et al 2011). In this paper, we investigate the macrobend-based sensing approach for developing stretch sensors, and apply this technology to pose sensing of a soft robotic arm.

The remainder of this paper is organized as follows. In Section 2, pose sensing based on macrobend stretch sensors is explained. This section also presents an analytical model which takes length values from three macrobend stretch sensors as inputs, shown in Figure 1, and delivers values of arm's length, bending angle, and pose of the arm's tip as outputs. The macrobend characterization experiments and the design and implementation of macrobend stretch sensors constitute Section 3. Section 4 presents the calibration and testing of the macrobend pose sensing system. The conclusions and future works are presented in Section 5.

\section{Pose sensing in a soft robotic arm}

The combination of position and orientation of an object, e.g. a soft robot arm, is referred to as the pose of that object (Akella and Mason 1998). Pose sensing is the task of determining the position and orientation of the object relative to a coordinate system. The pose sensing system, presented in the following, can be used to measure the pose information in human joints (e.g. wrist joint) as well as robotic systems (e.g. soft robotic arms). In this study, the sensing system is used for pose sensing of the STIFF-FLOP robot arm 
(Cianchetti et al 2013; Ranzani et al 2015a), shown in Figure 1. The body of this soft continuum arm is made of Silicone (Ecoflex 00-50, Smooth-on Inc.) and is $40 \mathrm{~mm}$ in length. The arm accommodates three longitudinal pneumatic chambers which are equally orientated around the central axis of the arm, and are constrained inside a crimped braided sleeve ${ }^{1}$. When the pneumatic chambers are inflated, the braided sleeve restrains the radial expansion of the chambers, thus guiding the ballooning effect to mainly occur along the longitudinal direction. We have developed custom sewable stretch sensors based on macrobend fibres used for length sensing in the peripheral edge of the arm; hence, the general term 'length sensor' is replaced by 'macrobend stretch sensor' in the following. The macrobend stretch sensors were sewn into the crimped braided sleeve of the soft robot arm, as shown in Figure 1.

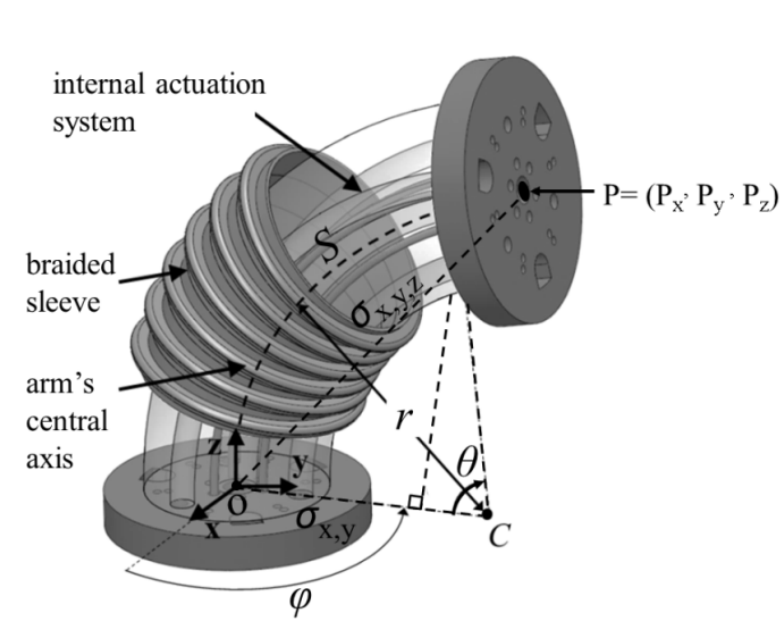

(a)

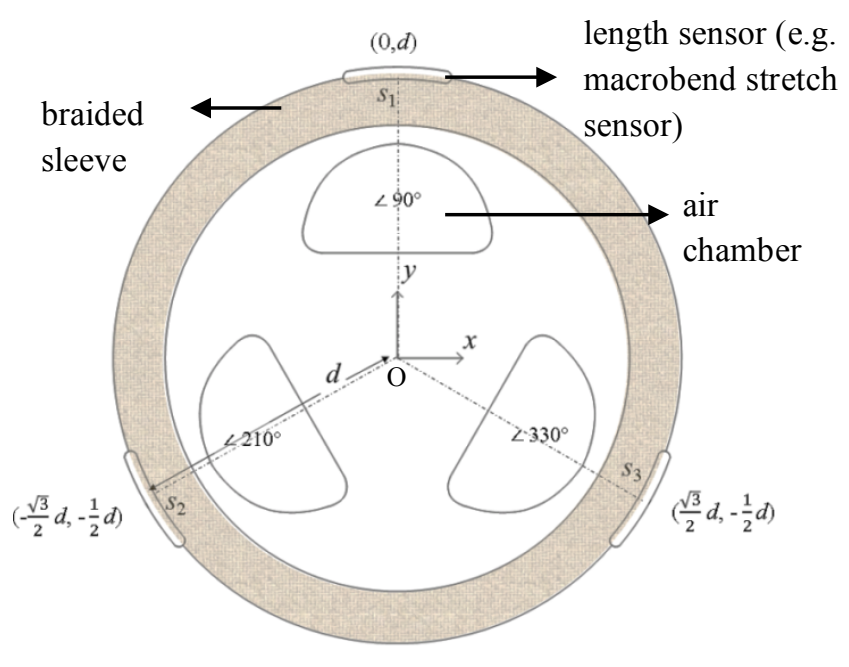

(b)

Figure 2. (a) Three dimensional shape parameters for a continuum robotic arm, (b) the cross-section view of the arm indicating the position of actuators (air chambers) and length sensors with lengths $s_{1}, s_{2}, s_{3}$.

The pose sensing system for the cylindrical arm considered here consists of three macrobend stretch sensors whose variable lengths are $s_{1}, s_{2}$, and $s_{3}$, respectively. In response to the amount and direction of bending, the three length sensors will be individually elongated or shortened. The combination of the three sensor outputs can then be used to compute the curvature parameters of the arm; i.e. length of the central axis of the arm (arm length) $S$, bending angle $\theta$, and orientation $\varphi$. In this study, these parameters are computed using the constant curvature model (Webster and Jones 2010). Figure 2a shows the parameters of the arm in 3D space. The frame of reference o-xyz is fixed at the base of the robot arm such that the xy-plane is the plane of the bottom of the arm and the z-axis is perpendicular to this plane. We assume that the arm bends with constant radius $r$. The arm length $S$ can be obtained as follows (Jones et al 2004),

$$
S=\frac{\left(s_{1}+s_{2}+s_{3}\right) d}{\sqrt{s_{1}^{2}+s_{2}^{2}+s_{3}^{2}-s_{1} s_{2}-s_{1} s_{3}-s_{2} s_{3}}} \sin ^{-1}\left(\frac{\sqrt{s_{1}^{2}+s_{2}^{2}+s_{3}^{2}-s_{1} s_{2}-s_{1} s_{3}-s_{2} s_{3}}}{3 d}\right)
$$

\footnotetext{
${ }^{1}$ Note that the crimping of the braided sleeve is performed to enhance its elongation range.
} 
The bending angle $\theta$ can be calculated as follows (Webster and Jones 2010),

$$
\theta=\frac{2 \sqrt{s_{1}^{2}+s_{2}^{2}+s_{3}^{2}-s_{1} s_{2}-s_{1} s_{3}-s_{2} s_{3}}}{3 d}
$$

where $d$ describes the radial distance between the central axis of the arm and each length sensor integrated at the peripheral edge. Finally, the orientation of the $\operatorname{arm} \varphi$ can be computed through (Webster and Jones 2010),

$$
\varphi=\tan ^{-1}\left(\frac{\sqrt{3}\left(s_{2}+s_{3}-2 s_{1}\right)}{3\left(s_{2}-s_{3}\right)}\right)
$$

Having calculated the values of the bending and orientation angles along with the length of the central axis of the arm, the tip position in Cartesian coordinates can be easily calculated from (Chen 2005),

$$
\mathrm{P}=\left(\mathrm{P}_{\mathrm{x}}, \mathrm{P}_{\mathrm{y}}, \mathrm{P}_{\mathrm{z}}\right)=\left\{\begin{array}{c}
\mathrm{Px}=\frac{\cos \varphi(1-\cos \theta)}{\theta} S \\
\mathrm{Py}=\frac{\cos \varphi(1-\cos \theta)}{\theta} S \\
\mathrm{Pz}=\frac{\sin \theta}{\theta} S
\end{array}\right.
$$

The capability of the sensing system in generating correct values of $S, \theta, \varphi$ can be evaluated using embedded position sensors at the tip and the base of the robotic arm, e.g. the Aurora V2 elecromagnetic tracking system (Northern Digital Inc., Canada). Assuning the base of the arm is located at the origin $(0,0,0)$, the bending radius of the arm can be calculated from the arm's tip position as follows (Malekzadeh et al 2014),

$$
r=\frac{\sigma_{\mathrm{x}, \mathrm{y}, \mathrm{z}}^{2}}{2 \sigma_{\mathrm{x}, \mathrm{y}}}
$$

where $\sigma_{\mathrm{x}, \mathrm{y}, \mathrm{z}}=\sqrt{P_{x}^{2}+P_{y}^{2}+P_{z}^{2}}$ is the length of the straight line connecting the base and the tip of the central axis of the arm (OP), and $\sigma_{\mathrm{x}, \mathrm{y}}=\sqrt{P_{x}^{2}+P_{y}^{2}}$ is its projection on the bottom plane of the arm. Using triangle rules, the angles $\theta$ and $\varphi$ can be expressed as,

$$
\begin{array}{r}
\theta=\cos ^{-1}\left(1-\frac{\sigma_{\mathrm{x}, \mathrm{y}}^{2}}{r}\right) \\
\varphi=\tan ^{-1}\left(\frac{P_{\mathrm{y}}}{P_{\mathrm{x}}}\right)
\end{array}
$$


Having computed the bending radius and the bending angle, the length of the central axis of the arm is obtained from,

$$
S=r \theta
$$

The position data from Aurora sensors are also required to be used as the reference for the initial calibration of the length sensors. The reference values of lengths $s_{1}, s_{2}, s_{3}$ can be calculated as (Jones 2007),

$$
\left\{\begin{array}{c}
s_{1}=S-\theta d \sin \varphi \\
s_{2}=S+\theta d \sin \left(\frac{\pi}{3}+\varphi\right) \\
s_{3}=S-\theta d \cos \left(\frac{\pi}{6}+\varphi\right)
\end{array}\right.
$$

Note that the orientation angle of the length sensors on the periphery of the arm with respect to $x$-axis are $90^{\circ}, 210^{\circ}$ and $330^{\circ}$, as indicated in Figure 2(b).

\section{Macrobend stretch sensing}

\subsection{Characterization of macrobend-induced light loss}

This section describes an experimental study concerning the proposed stretch sensors by exploiting the macrobend fibre concept (Silva et al 2013). Through this study, we aim to characterize the main parameters of the proposed stretch sensors: the bend fibre radius and the number of turns. The definition of an optical fibre turn with bend fibre radius $r$ is illustrated in Figure 3 (Belardi and Knight 2014).

(a)

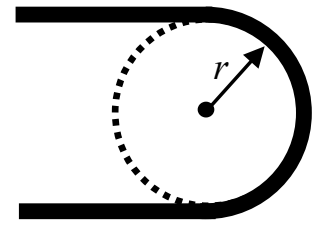

(b)

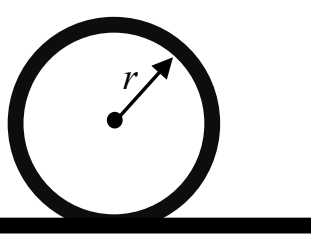

Figure 3. The definition of the optical fibre turn: a half-turn (a) vs. a turn (b), with bend fibre radius $r$. Note that the bend fibre radius is measured from the inside curvature.

In order to characterize the fibre macrobend, precise deployment (bending) of the optical fibre is required. The industry standard IEC 60793-1-47 (measurement methods and test procedures: macrobending) suggests two methods for the bending of optical fibres during macrobend loss measurement:

- Method A: Fibre winding, e.g., using mandrels.

- Method B: Quarter circle bends, e.g., using guiding groove structures on a flat surface.

Whilst Method A is the preferred way of bending for relatively large number of fibre turns, Method B is suitable for bending optical fibres in short lengths with relatively fewer bends. Therefore, in order to precisely modulate the light intensity through macrobending, a set of custom mandrels and guiding groove 
structures for the bending of optical fibres were designed and fabricated ${ }^{2}$. When the optical fibre is bent, the intensity of the modulated light is measured using an FS-N11MN fibre optic sensor (Keyence ${ }^{\mathrm{TM}}$, USA), which converts the light intensity to voltage. In order to characterize the macrobend loss, three sets of experiments were conducted:

Experiment 1. Characterization of the macrobend loss: planar bend patterns of a fibre formed inside guiding grooves: this experiment uses the guiding groove structures to form fractions of a turn $\mathrm{N}=\{1 / 8$, $2 / 8,3 / 8,4 / 8\}$ along a part of the length of the optical fibre. The experiment is conducted with three values of $r=\{5,3.5,2\} \mathrm{mm}$ for the bend fibre radius. Figure 4(a) shows a typical plastic guiding groove on the flat surface. Figure 4(b) shows the experimental results at various bend radii and the number of turns. It can be seen that the values of bend radius and the number of turns are both significantly influencing the light intensity for the number of turns $\mathrm{N}<4 / 8$.
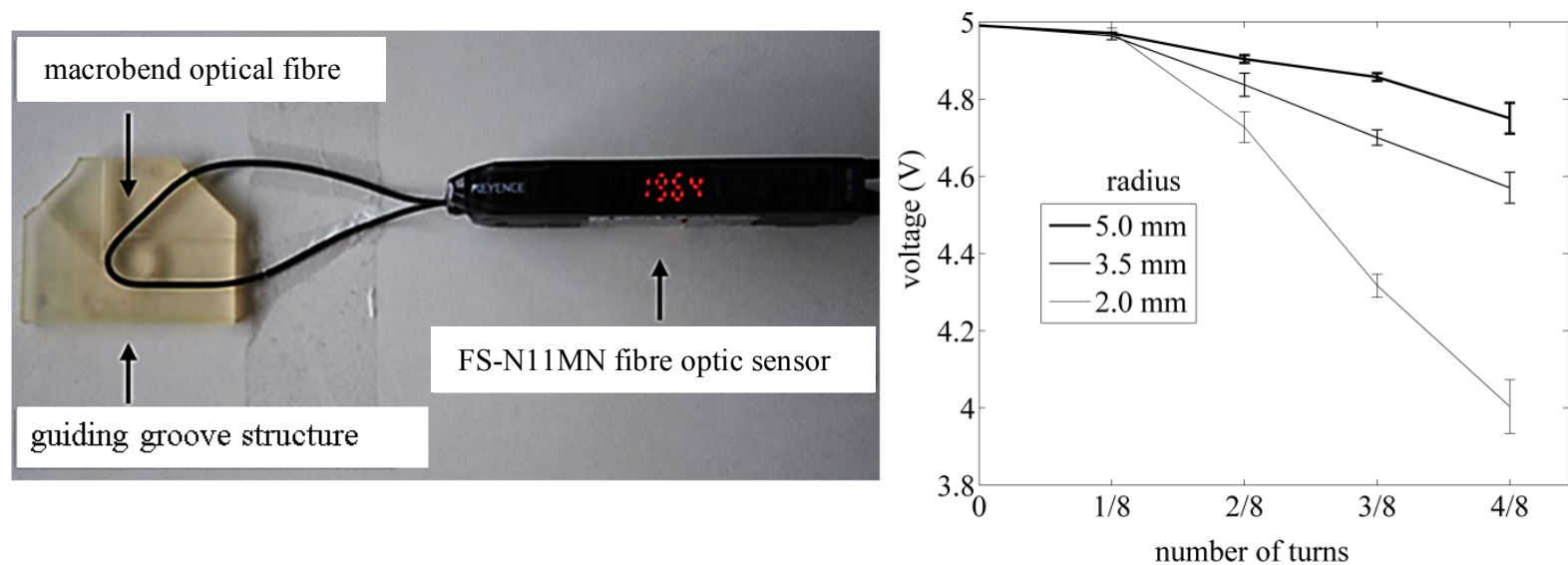

Figure 4. (a) The guiding groba structure forming a macrobent part $(r=3.5 \mathrm{~mm}$ and $\mathrm{n}=3(\mathrm{~b})$ along the length of the optical fibre (left) and the FS-N11MN fibre optic sensor (right), and (b) the change in voltage output when the number of turns increases at constant bend fibre radius.

Experiment 2. Characterization of the macrobend loss: planar bend patterns vs. 3D winded patterns: this experiment uses both groove and mandrel rigs, and is aimed to identify the difference in macrobend loss when equal number of turns are produced through forming inside successive guiding grooves (Figure 5(a)) vs. winding around a mandrel (Figure 5(b)). The experiment was performed for number of turns $N=\{1,2$, 3 \}. The experimental results (Figure 5(c)) imply that the sensing voltage depends on the bend fibre radius and the number of turns in both cases, i.e. there are approximately equal sensing values (maximum difference of $3 \%$ ) for two successive half turns using guiding grooves and a turn using mandrels. Therefore,

\footnotetext{
${ }^{2}$ In this study, the SH1001-1.0 Super Eska ${ }^{\mathrm{TM}}$ Polyethylene jacketed optical fiber cord (Mitsubishi Rayon Co., Ltd., Japan) with a core diameter of $217-263 \mu$ and weight of $0.74 \mathrm{~g} / \mathrm{m}$ has been used for experimentation. The core and cladding materials are polymethyl-methacrylate resin and fluorinated polymer, respectively. The core reflective index for this fiber is 1.49 and according to the datasheet of the fiber, no deterioration in optical properties of this fiber occurs while operating under $60^{\circ} \mathrm{C}$. The guiding groove structures were designed in SolidWorks 3D CAD software (SolidWorks Corp.) and fabricated from Visijet EX200 via a Projet HD 3000 3-D production system.
} 
we can calculate the macrobend loss in complex helical or 2D patterns of mutually-tangent circular arcs only through counting the number of turns and associated radii.

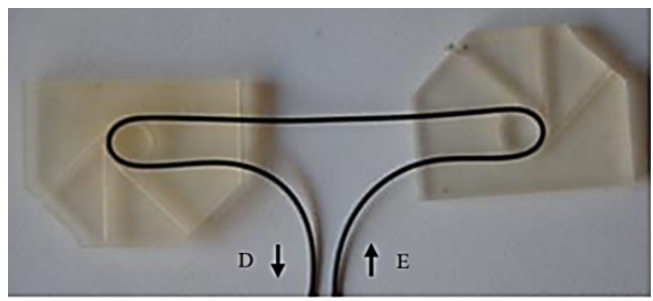

(a)

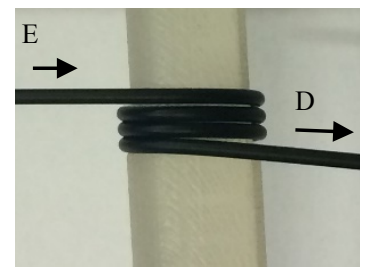

(b)

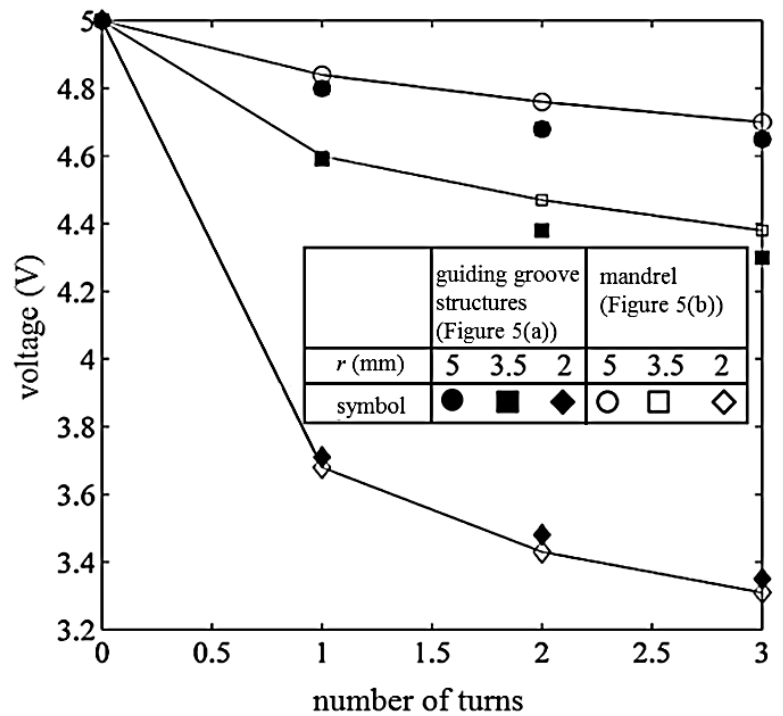

(c)

Figure 5. The experimental configuration for producing macrobend loss through (a) shaping the fibre using guiding groove structures (one turn), and (b) winding the fibre around a mandrel (three turns). (c) The experimental data which indicates bending the fibre through two abovementioned approaches achieve virtually the same results (voltage values). The arrows show the light input and output directions. Note that ' $E$ ' and ' $D$ ' indicate the connections to the FS-N11MN light emitter and detector, respectively.

Experiment 3. Characterization of the macrobend loss: 3D winded patterns: this experiment uses mandrels of various cross-section radii $r=\{2,2.5,3,3.5,4,4.5,5,5.5,6,6.5,7\} \mathrm{mm}$ for winding multiple turns $\mathrm{N}=$ $\{1, \ldots, 10\}$ of optical fibres. As the number of turns is increased, the sensing voltage varies as shown in Figure 6(a). Note that the measurements of the voltage were taken every half a turn, hence we have 21 measurement points per radius. There is a clear elbow in each constant radius graph where the number of turns is 5 . After this point, the rate of reduction in voltage values is very small, e.g. a maximum change of $4 \%$ in the voltage values was measured whilst increasing the number of turns from $\mathrm{N}=5$ to $\mathrm{N}=10$ across different radii. In order to further explain this observation, the standard deviation of the last 11 samples of voltage at each radius was calculated and then divided by the standard deviation of the all 21 samples at each radius to result in a normalized standard deviation (NSD) expressed as

$$
\mathrm{NSD}=\frac{\text { SD of last } 11 \text { voltage samples at constant radius } r}{\text { SD of all voltage samples at constant radius } r}=\frac{\sqrt{\frac{\sum_{i=11}^{21}\left(v_{i, r}-\eta_{r}\right)^{2}}{11}}}{\sqrt{\frac{\sum_{i=0}^{21}\left(v_{i, r}-\mu_{r}\right)^{2}}{20}}}
$$

where $v_{i, r}$ is the voltage value corresponding to the sample number $i=\{1, \ldots, 21\}$ and the bending radii $r=$ $\{0.5,1,1.5,2,2.5,3,3.5,4,4.5,5,5.5,6,6.5,7\}$. The $\eta_{r}$ and $\mu_{r}$ represent average voltage values at constant radius $r$, 


$$
\eta_{r}=\frac{1}{11} \sum_{i=11}^{21} v_{i, r}, \mu_{r}=\frac{1}{21} \sum_{i=0}^{21} v_{i, r}
$$

Table 1 shows that the absolute standard deviation of the last 11 voltage samples at each radius is less than $0.04 \mathrm{~V}$. Also, the Figure 6(b) shows that the ratio of absolute deviation of the last 11 samples and the standard deviation of all 21 samples significantly decrease by increasing the bend fibre radius. Hence, the dependence of the voltage to the number of turns can be assumed negligible when the number of turns is equal or greater than 5. This assumption simplifies designing sensing systems based on macrobend loss, taking into account the bend radius as the only significantly influencing design parameter. We therefore focus our work on the design and implementation of a stretch sensor with number of turns equal or greater than 5 .

In general, the light loss in an optical fibre happens when the radius of bending is below a specific threshold called the Minimum Bending Radius (MBR). This value is a function of the material properties, thickness, and also the number of turns (Hoss and Lacy 1993). The MBR for a quarter turn of the fibre used in this study is $5 \mathrm{~mm}$, according to its datasheet. Referring to the experimental data presented in Figure 4(b), a quarter bend can produce $0.1 \mathrm{~V}$ drop in the sensing voltage. Clearly, increasing the number of turns causes drop in sensing voltage and, hence, raises the value of the MBR. Here, we are interested in designing macrobend sensors with $\mathrm{N} \geq 5$. According to the experimental measurements, presented in Figure 6(a), a $0.1 \mathrm{~V}$ voltage drop can occur by bending under the radius of $6.5 \mathrm{~mm}$ when $\mathrm{N} \geq 5$. Therefore, we choose $\mathrm{MBR}=6.5 \mathrm{~mm}$ for the proposed stretch sensor.

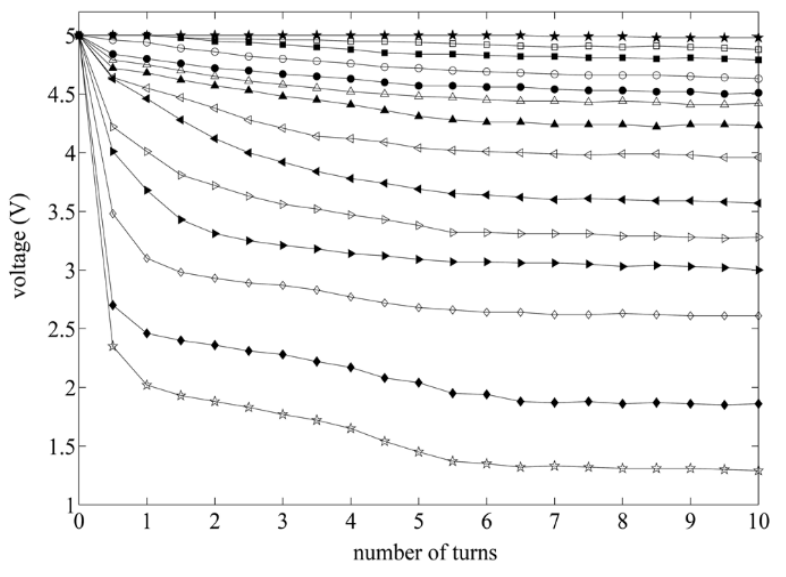

(a)
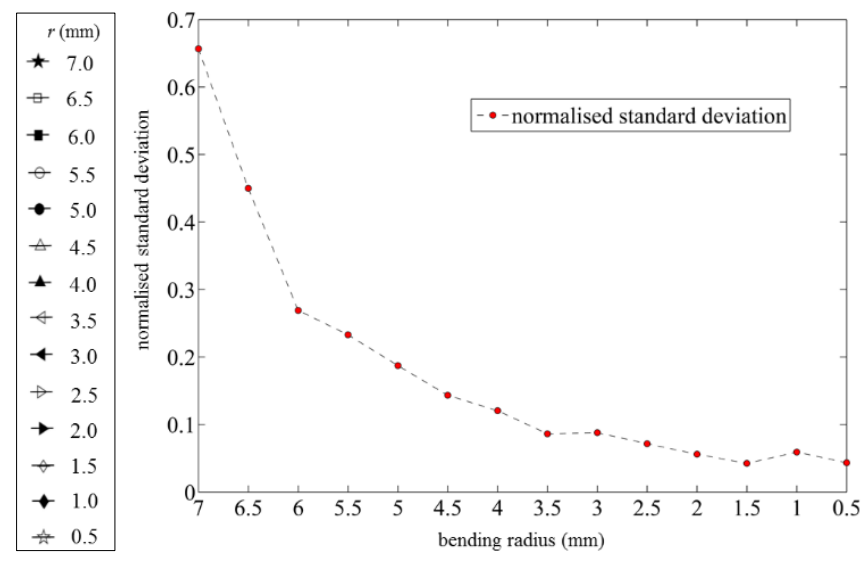

(b) 
Figure 6. (a) The FS-N11MN analogue output voltage as the number of macrobend turns around the mandrel is increased, (b) the radio of standard deviation of last 11 samples and the standard deviation of all 21 samples significantly decreases by increasing the bend fibre radius.

Table 1. The absolute standard deviation of last 11 voltage samples at each bend fibre radius.

\begin{tabular}{|l|l|l|l|l|l|l|l|l|l|l|l|l|l|l|}
\hline $\mathbf{r}(\mathbf{m m})$ & $\mathbf{7 . 0}$ & $\mathbf{6 . 5}$ & $\mathbf{6 . 0}$ & $\mathbf{5 . 5}$ & $\mathbf{5 . 0}$ & $\mathbf{4 . 5}$ & $\mathbf{4 . 0}$ & $\mathbf{3 . 5}$ & $\mathbf{3 . 0}$ & $\mathbf{2 . 5}$ & $\mathbf{2 . 0}$ & $\mathbf{1 . 5}$ & $\mathbf{1 . 0}$ & $\mathbf{0 . 5}$ \\
\hline SD (V) & 0.03 & 0.04 & 0.02 & 0.03 & 0.03 & 0.04 & 0.02 & 0.03 & 0.02 & 0.02 & 0.03 & 0.02 & 0.02 & 0.01 \\
\hline
\end{tabular}

\subsection{Design of the stretch sensor}

The macrobend stretch sensor should be highly compact at compression, and stretchable to a particular length required by the actuator it is coupled with to measure its length change. Also, in order to keep all electronics away from the sensing site, the fibre should be looped to place the light emitter and detector next to each other. Figure 7(a) illustrates the loopback design of the macrobend stretch sensor indicating locations of tip vertices, lateral vertices, and fibre cross-overs. Note that during a full stretch of the sensor, the radius of curvature of lateral vertices are increased resulting in an increment in the light intensity, whilst the radius of curvature at the tip vertex is decreased generating an inverse effect on the light intensity value. Hence, the voltage output is not valid when the bend fibre radius at the tip vertex is less than the MBR of the optical fibre $(r<6.5 \mathrm{~mm})$. In order to avoid this problem, the radius of curvature at the tip vertex must be always kept above the MBR, so that this vertex is not used for sensing.

Figure 7(b) shows three key phases of stretch sensing, each comprised of a chain of ellipsis, where $a$ and $b$ represent semi major axes of each ellipse. Each ellipse has two lateral vertices that can be designed to produce certain levels of light loss at certain stretch levels of the sensor structure. The levels of light loss depend on how the bend fibre radius of lateral vertices is changing in relation with the MBR. We assume that the perimeter of each ellipse remains constant during the length change of the sensor structure, which means there is no exchange of fibre length at fibre cross-over locations during the operation of the sensor. Hence, knowing the number of ellipses $\mathrm{N}$ and the bend fibre radius of the balanced configuration $\left(r_{\text {balance }}\right)$, a unique sensor structure can be designed. Table 2 summarizes the impact of the choice of $r_{\text {balance }}$ on the compactness and stretchability of the sensor, suggesting that $b_{\max }=r_{\text {balance }}=$ MBR can be considered as a reasonable trade-off in the design of the sensor. Note that $b_{\max }$ is regarded as a measure for the stretchability of the sensor, and $w_{\max }$ increases with $b_{\max }$ and represents the maximum value of the semi major axis $b$. Also, according to the experimental results presented in Figure 6(a), the voltage value is $1.35 \mathrm{~V}$ at a bend fibre radius of $0.5 \mathrm{~mm}$ (this value was measured by winding the fibre around a still needle with the diameter of $1 \mathrm{~mm})$. The $b=0$ cannot be implemented and tested using mandrels. We consider $b_{\min }=0.5 \mathrm{~mm}$ to allow sufficient voltage margin with the radius range that cannot be tested through this approach, where $b_{\min }$ is the minimum value of the semi major axis $b$.

This study aims to integrate the abovementioned macrobend stretch sensor into the STIFF-FLOP arm for pose measurement. The three macrobend stretch sensors should be installed at specified locations along the periphery of the arm (Figure 2(b)). Therefore we need information on the maximum stretch at these locations. The maximum applicable air pressure inside pneumatic chambers of the STIFF-FLOP arm (Figure 1) is 0.6 bar; beyond this amount of pressure the robot arm is likely to be damaged. Therefore, an 
air chamber of the arm was inflated by 0.6 bar, undergoing a $163^{\circ}$ bending deformation in one direction. This actuation increased the length of the central axis of the arm from $40 \mathrm{~mm}$ to $83 \mathrm{~mm}$, as presented in Figure 8(a).

(a)

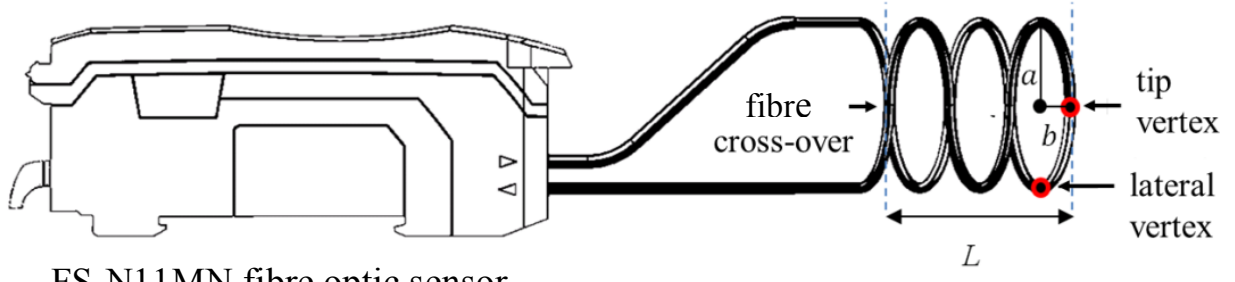

(b)

FS-N11MN fibre optic sensor

\begin{tabular}{|l|l|l|}
\hline Sensing phase & \multicolumn{1}{|c|}{ Description } \\
\hline 1.compression & & \\
\hline 2.balance & (D) & \\
\hline 3.extension & (E) & \\
& &
\end{tabular}

Figure 7. (a) The loopback configuration of the macrobend stretch sensor, (b) the three key phases of sensing including compression, balance, and extension.

According to the Equation (9), in order to calculate the maximum length along the periphery of the arm, we need to know the cross-section radius of the braided sleeve, indicated in Figure 2(b). The value of $d$, decreases in response to the elongation of the arm. Therefore, the relationship between the length and crosssection radius of the crimped braided sleeve was studied and the results are shown in Figure 8(b). It can be seen that the cross-section radius is $16.5 \mathrm{~mm}$ for an arm length of $40 \mathrm{~mm}$. This value decreases to $14.5 \mathrm{~mm}$ at maximum elongation of $83 \mathrm{~mm}$ (this value of length corresponds to a bending angle of $163^{\circ}$ during actuation, according to the bending angle vs. length data presented in Figure 8(a)). The linear fit to the measured length vs. cross-section data was calculated in Matlab as, 


$$
d=-0.047 S+18.4
$$

Table 2. The choices of the balance bend fibre radius $r$ balance and their impact on stretchability and compactness of the sensor structure.

\begin{tabular}{|c|c|c|c|c|c|}
\hline \multirow{2}{*}{$r_{\text {balance }}$} & \multicolumn{3}{|c|}{ Configuration involved } & \multirow{2}{*}{$\begin{array}{c}\text { Stretchability } \\
\text { (measure: } \boldsymbol{b}_{\max } \text { ) }\end{array}$} & \multirow{2}{*}{$\begin{array}{l}\text { Compactness } \\
\text { (measure: } \boldsymbol{w}_{\max } \text { ) }\end{array}$} \\
\hline & compression & balance & extension & & \\
\hline $\boldsymbol{r}_{\text {balance }}<\mathrm{MBR}$ & yes & no & no & low & high \\
\hline $\boldsymbol{r}_{\text {balance }}=$ MBR & yes & yes & no & medium & medium \\
\hline $\boldsymbol{r}_{\text {balance }}>\mathrm{MBR}$ & yes & yes & yes & high & low \\
\hline
\end{tabular}
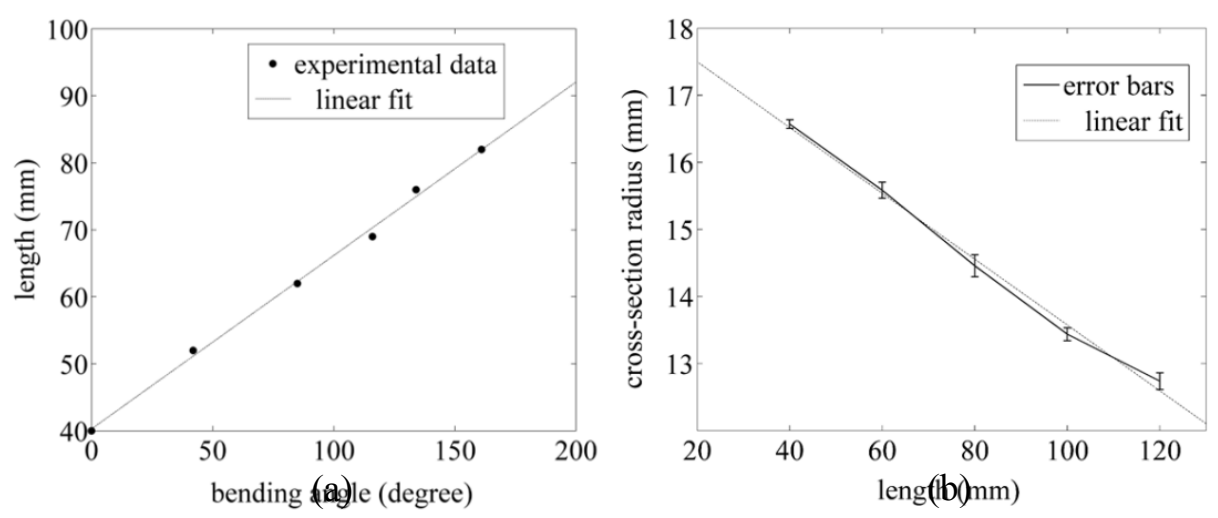

Figure 8. The length and cross-section radius of the STIFF-FLOP arm are functions of its bending angle: (a) the length vs. bending angle, and (b) the cross-section radius vs. length.

Under the assumption of constant curvature bending, the radius of curvature of the arm under maximum pressurization of 0.6 bar is $r=\frac{83}{\theta}=29.2 \mathrm{~mm}$ where $\theta=163^{\circ}$ is the bending angle. The minimum radius of the arm's circular cross-section is $d_{\text {min }}=14.5 \mathrm{~mm}$. Therefore, the maximum lateral length $L_{\max }=(r+$ $\left.d_{\min }\right) \theta$ is calculated as $L_{\max }=124.3 \mathrm{~mm}$. Referring to the Figure 7(a), the number of ellipsis can be calculated through,

$$
N=\frac{L-2 \mathrm{~N} b}{\mathrm{f}}-1
$$

By substituting quantities of $L=L_{\max }=127 \mathrm{~mm}, b=b_{\max }=6.5 \mathrm{~mm}$, and $\mathrm{f}=1 \mathrm{~mm}$ into the Equation (13), the number of ellipsis (fibre turns) is computed as $\mathrm{N}=9$. It is worth reiterating that the maximum length measured in the robot arm is $124.3 \mathrm{~mm}$, hence $L_{\max }=127 \mathrm{~mm}$ provides around $3 \mathrm{~mm}$ margin above this value. Having substituted $\mathrm{N}=9$, and $b=b_{\min }=0.5 \mathrm{~mm}$ in the same equation gives the minimum measurable 
length by this sensor as $L=L_{\min }=19 \mathrm{~mm}$. Therefore, the stretch sensor is able to measure lengths between $19 \mathrm{~mm}$ to $127 \mathrm{~mm}$.

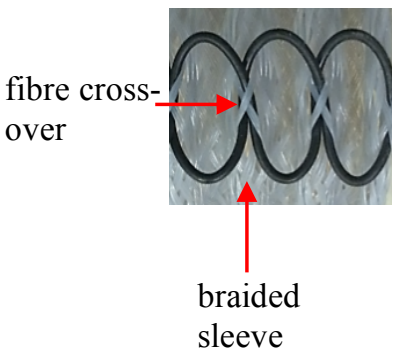

(a)

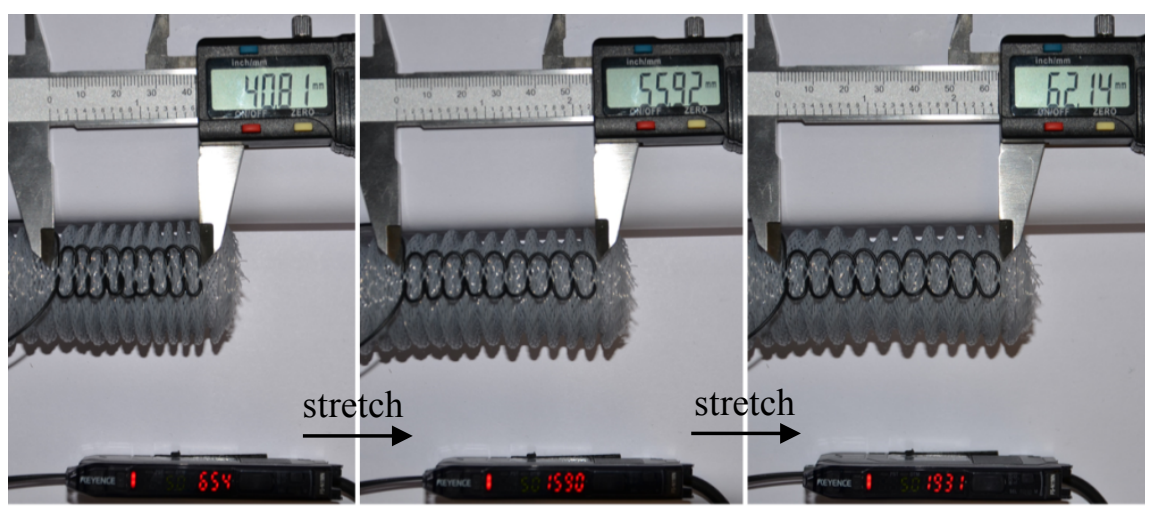

(b)

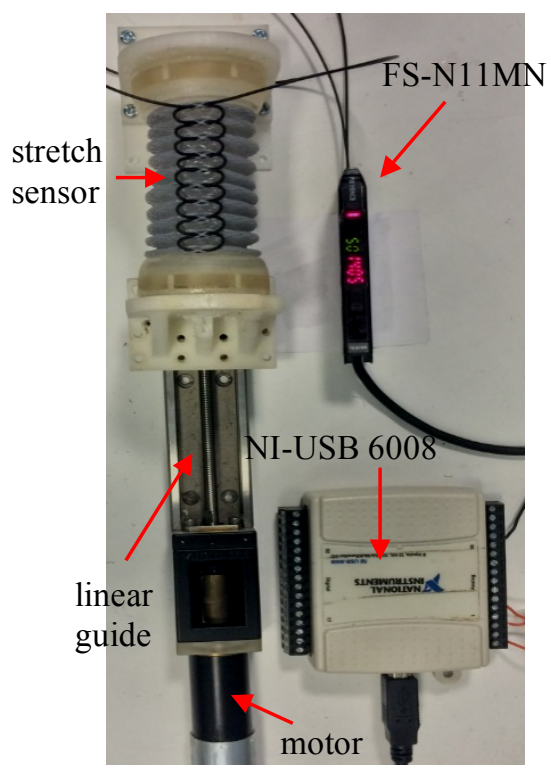

(c)

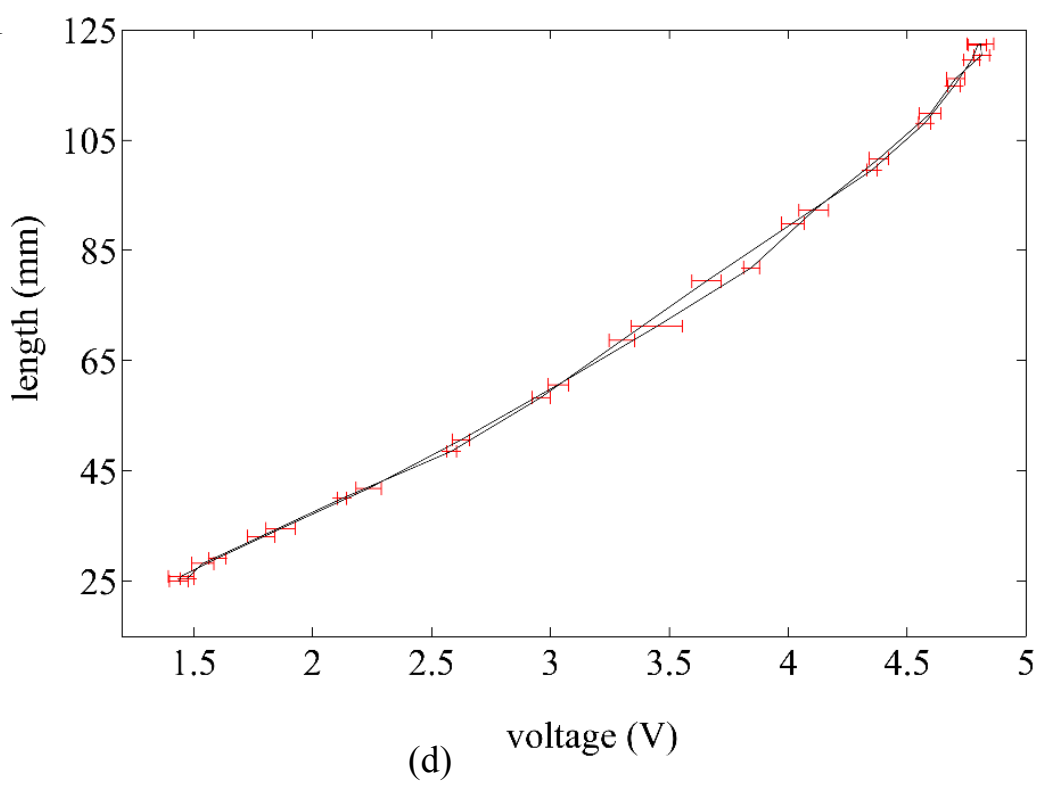

Figure 9. (a) Close-up view of the braided sleeve with an integrated stretch sensor, (b) Configuration of the macrobend stretch sensor integrated into a crimped braided sleeve at three different stretch levels. The FS-N11MN fibre optic sensor displays the light intensity values corresponding to the lengths measured by a digital caliper, (c) the experimental test rig used to characterize the macrobend stretch sensor. A braided sleeve with an integrated stretch sensor was fixed between the fixed and moveable platforms of a linear actuator; the voltage data from FS-N11MN 
fibre optic sensor and motor position were synchronously acquired during the test. (d) Test results: the length-voltage relationship of the stretch sensor.

Note that $\mathrm{N}=9$ is safely satisfying the optical condition $(\mathrm{N} \geq 5)$ for relying only on the radius of curvature in calculation of the macrobend loss, discussed in section 3.1 (Experiment 3) and Figure 6(a) and 6(b). In order to experimentally validate the sensor design, the optical fibre was sewn according to the loopback configuration shown in Figure 9(a) and 9(b) into a crimped braided sleeve $\mathrm{e}^{3}(\mathrm{~N}=9$ and $r$ balance $=6.5 \mathrm{~mm})$. By stretching the braided sleeve structure, the macrobend sensing system is expected to be continuously modified from highly compressed to the balanced configuration, ideally providing a sensing range of $1-5 \mathrm{~V}$ (the measurable range by FS-N11MN fibre optic sensor). Figure 9(c) shows the experimental setup of a test where the stretch sensor was subjected to pure elongation along the $\mathrm{x}$-axis, using a linear actuator. The voltage output of the FS-N11MN fibre optic sensor and the motor position were synchronously acquired during the test. This characterization test was run for 6 cycles and the sensor's voltage-length relationship with errorbars is presented in Figure 9(d). In the following, we report on our pose sensing system employing three macrobend stretch sensors of the type presented above.

We have performed a force test to quantify the stiffness of the macrobend pose sensor, as shown in Figure 10(a). In this experiment, a Nano17 force sensor anchored to the moving platform of a motorized linear guide was serially linked to the macrobend pose sensor. This experimental arrangement provided position and force information during the extension of compression of the pose sensor, as shown in Figure 10(b).

(a)

ATI Nano 17 force/ torque sensor

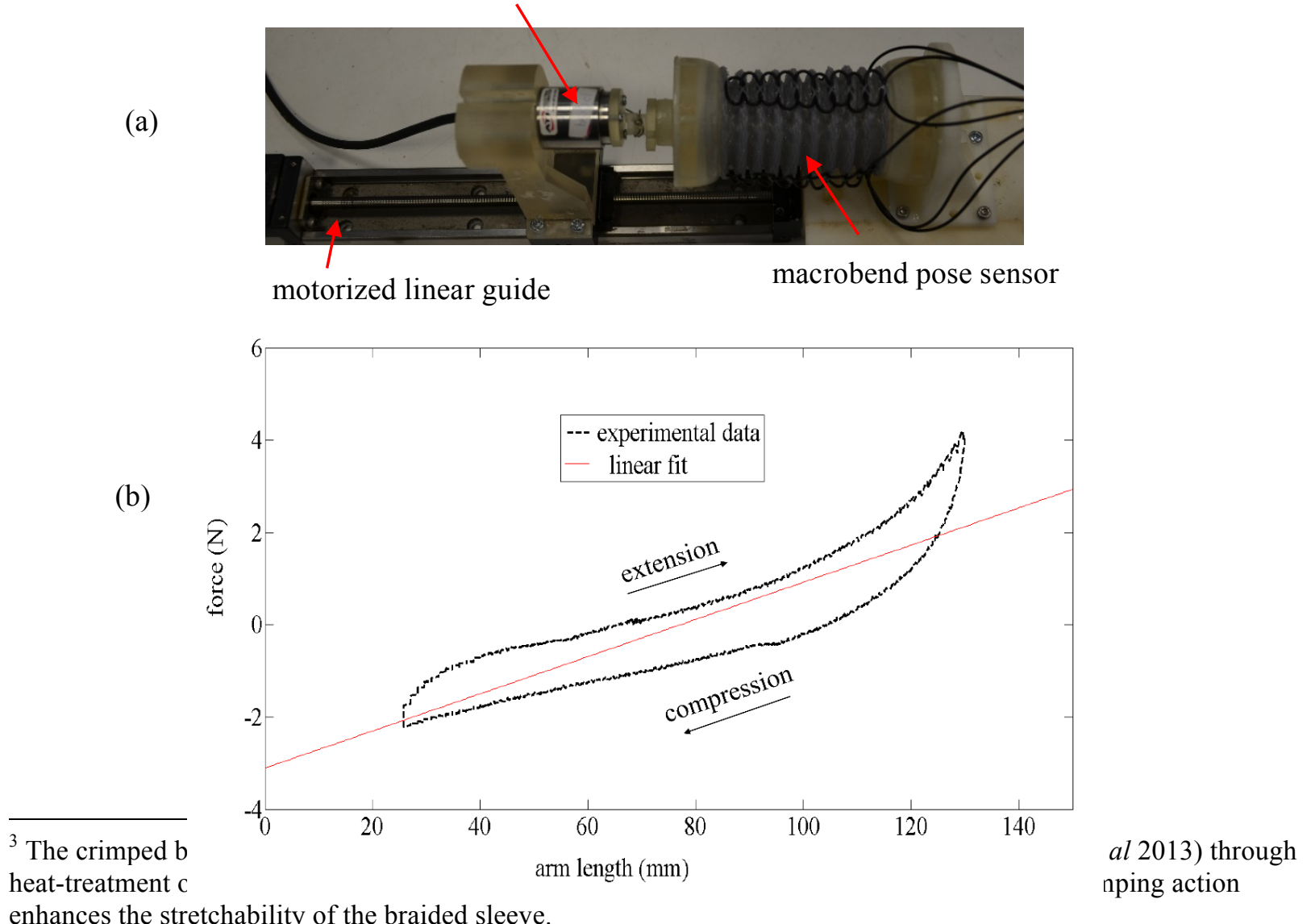


Figure 10. Sensor stiffness measurement: (a) the experimental setup, and (b) experimental results. Note that a Nano17 $\mathrm{F} / \mathrm{T}$ sensor is linked with the macrobend pose sensor.

The experimental results show that the maximum stiffness coefficient of the pose sensor (three stretch sensors integrated into the braided sleeve) is approximately $0.06 \mathrm{~N} / \mathrm{mm}$. The stiffness coefficient of the STIFF-FLOP arm was calculated as $3.05 \pm 0.11 \mathrm{~N} / \mathrm{mm}$ by Ranzani et al $2015 \mathrm{~b}$. Hence, the stiffness of the actuator is approximately 51 times bigger than the maximum stiffness of the pose sensor.

\section{Calibration and testing of the pose sensing system}

In order to experimentally validate the capability of the sensing system in measuring the pose of the soft robotic arm, the values of the configuration parameters obtained from the macrobend pose sensing system was benchmarked against the an electromagnetic tracking system (NDI Aurora). For this purpose, two NDI Aurora Micro 6DOF sensors (each $0.8 \mathrm{~mm}$ in diameter and $9 \mathrm{~mm}$ in length) were embedded in the STIFFFLOP robot arm with the integrated macrobend stretch sensors; one at the tip and the other one at the base of the arm (Figure 11(a)). The robot arm was actuated through pressurization of the pneumatic chambers using ITV0030-3BS-Q pressure regulators (SMC Corporation, USA) and a bench air compressor. Three swept-amplitude sine waves signals with the same frequency of $0.1 \mathrm{~Hz}$, a mutual phase difference of $120^{\circ}$, and linearly varying amplitude of 0-0.6bar (Figure 11(b)) were applied to the pressure regulators to move the arm in the $3 \mathrm{D}$ space. A custom $\mathrm{C \#}$ code was developed to synchronously acquire the analogue values from the serial port used by the NDI Aurora sensor and a USB port connected to FS-N11MN fibre optic sensors via a NI-USB 6211 DAQ. The position information from the NDI Aurora sensors and voltage data from the FS-N11MN fibre optic sensors connected to macrobend stretch sensors were synchronously acquired during the actuation. The position readings from the tip and base NDI Aurora sensors were translated in 3D space, with the base of the arm located on the origin $(0,0,0)$. Then, the translated tip positions were substituted into Equations (5) to (9) to eventually produce the length data for each macrobend stretch sensor. The resulting length data were curve-fitted against their corresponding voltage data from the FS-N11MN fibre optic sensors using a two-term exponential function of the form,

$$
s_{i}=\mathrm{c}_{1} e^{\mathrm{c}_{2} v_{i}}+\mathrm{c}_{3} e^{\mathrm{c}_{4} v_{i}}
$$

The coefficients $c_{n}, n=1,2,3,4$ were calculated in Matlab and is presented in Table 3. Figure 11(c) shows the voltage-length relationships of the macrobend stretch sensors and the corresponding two-term exponential fits. In order to validate the sensing values, the swept-amplitude sine waves were reapplied to the pressure regulators. The NDI Aurora sensors were to read the tip and base position data, and simultaneously the voltage data from FS-N11MN optical fibre sensors were acquired. The FS-N11MN output voltage values $v_{\mathrm{i}}$, where $i$ is the voltage sample number, were used as the input value for Equation (14) in order to calculate the associated length values $s_{\mathrm{i}}$. These length values were substituted into Equations (1) to (3) to obtain the length of the arm, the bending angle, orientation and position of the arm's tip. The reference values of the length of the arm, bending angle and orientation were calculated from reference position values provided by NDI Aurora sensors using Equations (5) to (8). Note that the position accuracy 
of the 6-DOF NDI Aurora sensors is claimed to be $0.90 \mathrm{~mm}$ (Nafis et al 2006). A comparison between sensing data from macrobend and NDI Aurora sensors is presented in Figures 12, and 13.

Table 3. The calculated values of coefficients $\mathrm{c}_{\mathrm{i}}, \mathrm{i}=1,2,3,4$ of the two-term exponential fit to voltage-length relationships of stretch sensors with lengths $s_{1}, s_{2}, s_{3}$.

\begin{tabular}{|l|l|l|l|l|}
\hline & $\mathbf{c}_{\mathbf{1}}$ & $\mathbf{c}_{\mathbf{2}}$ & $\mathbf{c}_{\mathbf{3}}$ & $\mathbf{c}_{\mathbf{4}}$ \\
\hline $\boldsymbol{s}_{\mathbf{1}}$ & 25.331 & -1.770 & 10.582 & 0.518 \\
\hline $\boldsymbol{s}_{\mathbf{2}}$ & 23.136 & 0.118 & 1.332 & 0.883 \\
\hline $\boldsymbol{s}_{\mathbf{3}}$ & 12.588 & -0.012 & 4.321 & 0.668 \\
\hline
\end{tabular}

(a)

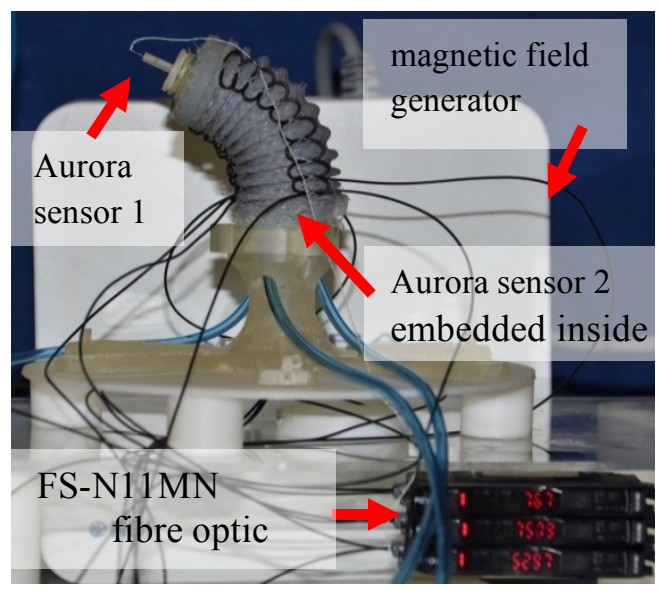

(b)

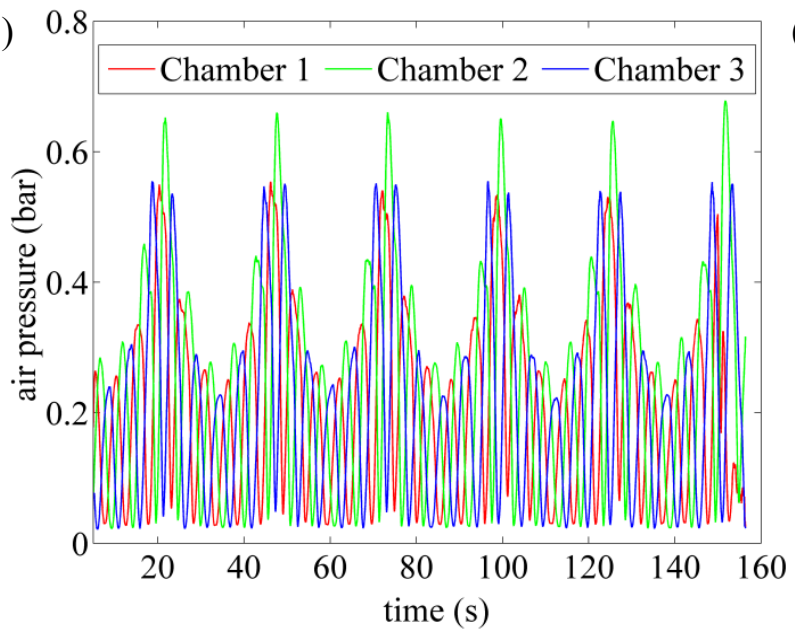

(c)

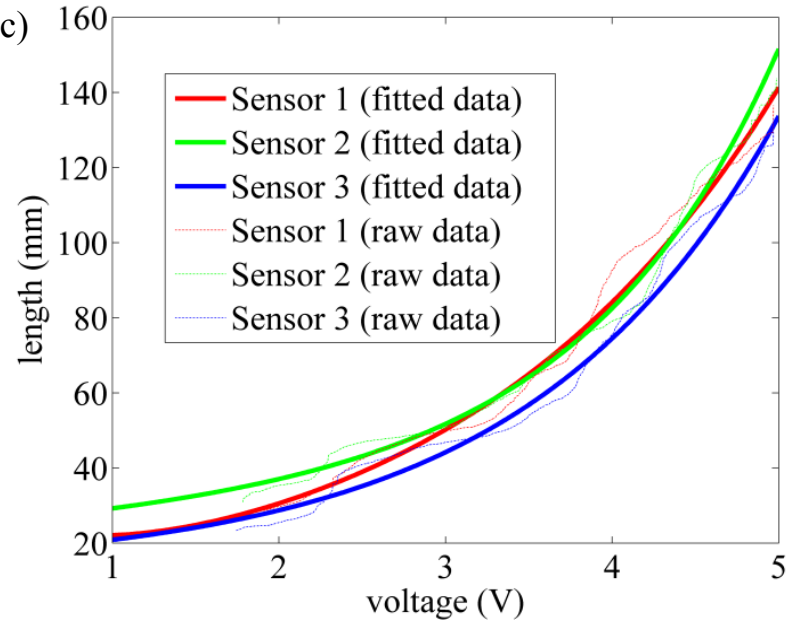

Figure 10. (a) The macrobend pose sensor's benchmarking setup with two NDI Aurora sensors at the base and the tip of the soft arm, sewn macrobend stretch sensors and FS-N11MN fibre optic sensors, (b) the swept-amplitude input signal which actuates the arm in 3D space, , and (c) the voltage-length relationships of the macrobend stretch sensors and the corresponding two-term exponential fits.

Figures 12 (a) and (b), present values of arm length and bending angle obtained from the macrobend pose sensor and the NDI Aurora sensors. The results of benchmarking experiments indicates that the pose sensor 
is capable of measuring the arm length and bending angle with maximum errors of $7 \mathrm{~mm}(18 \%)$ and $14^{\circ}(13 \%)$, respectively.

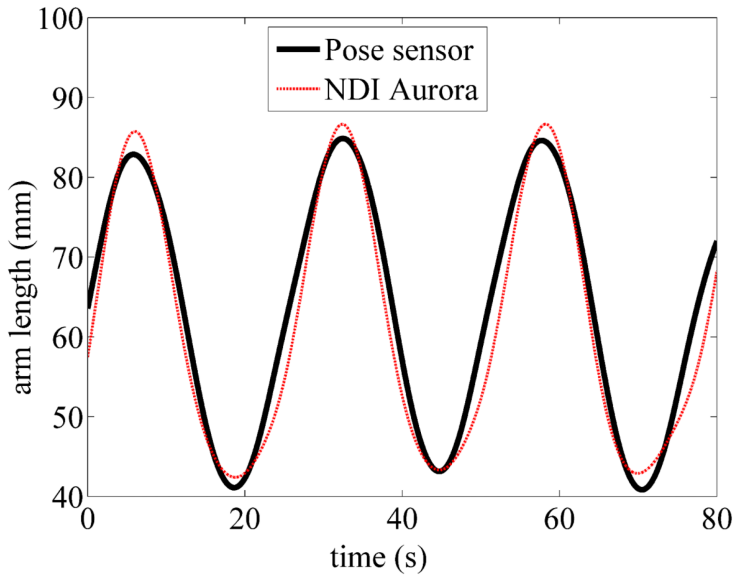

(a)

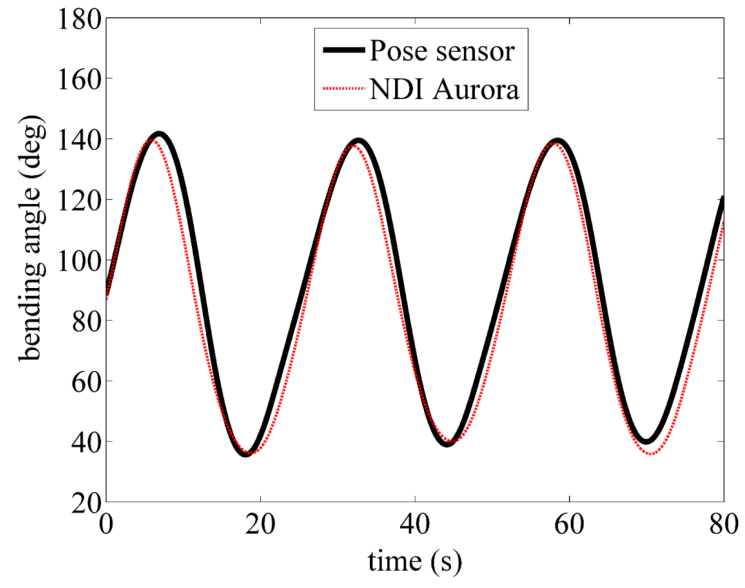

(b)

Figure 12. The comparison between the configuration parameters obtained from the macrobend pose sensor and NDI Aurora sensors: (a) the arm length, and (b) the bending angle.

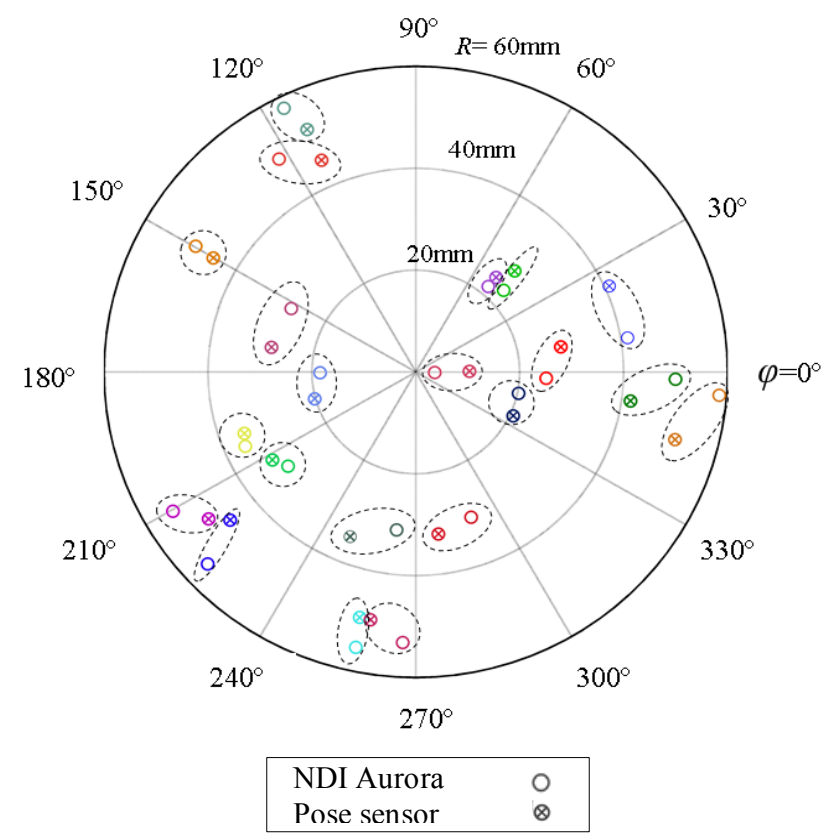

(a)

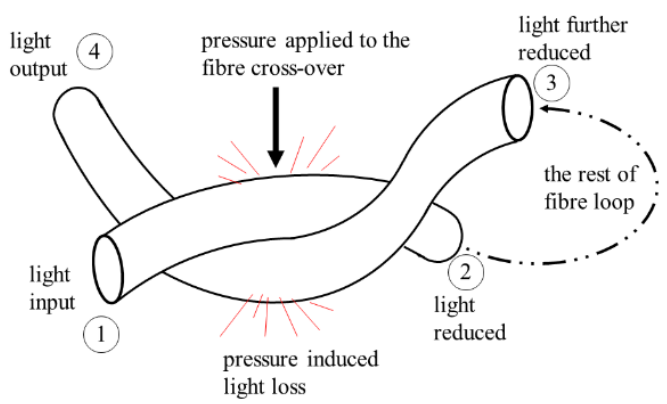

(b)

Figure 11. (a) The pose information obtained from the macrobend pose sensor and relevant reference data from NDI Aurora sensors. Note that dashed ellipsis show pairs of relevant data from macrobend pose and NDI Aurora. (b) The pressure induced light loss at fibre cross-over locations.

The repeatability of the sensing values defined as, 


$$
\text { Repeatability }=\frac{\text { standard deviation in repeated measurements }}{\text { sensing range }} \%
$$

was calculated for the bending angle (across 5 actuation cycles of swept-amplitude signal) as $94.8 \%$. Note that the standards deviation was calculated in Matlab as $5.6^{\circ}$.

Figure 13 presents the projection of the tip position on the bottom plane (xy-plane) of the robot arm, $\mathrm{P}(R, \varphi)$, where $R=\sigma_{\mathrm{x}, \mathrm{y}}$ (described in Section 2) and $\varphi$ is the orientation angle. The maximum error in the estimation of the projected position of the arm's tip on the xy-plane (shown in

Figure 11(a)) is $9.7 \mathrm{~mm}$. The error in the estimation of the configuration parameters can be due to the following reasons:

- The mismatch between the actual configuration of the arm and the ideal constant curvature shape assumption. The characterization experiments of the actuation system indicates a maximum deviation of $4 \%$ with respect to the constant curvature model (Ranzani et al 2015a).

- Also, the stress generated at the fibre at cross-over locations due to bending deformation of the arm, can change the intensity of the light received by detector (Wang et al 2005), and was not considered in the current model. This phenomenon, illustrated in

- Figure 11(b), can be observed in Figure 12(a), where the minimum and maximum values of length obtained from the pose sensor are below associated values from the NDI Aurora sensors.

This study has used a simple but established model for the analysis of a novel sensing system, however, future work on the stretch sensor will need to consider improvements on this model to reduce the error. The new model will ideally take into account the variable curvature of the robot arm and the light-loss at fibre cross-over locations. The improvement on the model can be achieved by introducing experimentally derived correction terms into the existing model to achieve a better fit.

This paper offers a solution to pose sensing in soft robotic arms using the proposed macrobend technique which is particularly cost-effective when compared with alternative solutions such as those based on FBG or electromagnetic tracking systems. Our stretch sensing system uses three Keyence FS-N11MN fibreoptic sensors which can currently be obtained for less than 750 USD overall. The used optical fibres are negligible cheap at less than 5 USD per meter. Approaches based on FBG or electromagnetic tracking systems are easily a magnitude more expensive.

\section{Conclusions}

We have presented a new pose sensing system, based on integrating a set of custom stretch sensors, and assessed its capabilities in measuring bending, elongation and compression in a soft robotic arm through benchmarking against an electromagnetic tracking system (NDI Aurora). The results of experiments indicated maximum errors of $18 \%$ and $13 \%$ in measuring the arm's length and the bending angle, respectively. The stretch sensors developed as a part of this study are made from highly flexible optical fibres. The measurable light outputs passing through the optical fibres is varies due to macrobend loss, 
which is a function of the fibre bend radius as well as the number of repeated turns. Our experimental study revealed that the chosen bend radius $r$ has a far greater impact on the measured light intensity values than the number of turns $\mathrm{N}$ (if greater than five). Hence, taking into account that the bend radius is the only significantly influencing design parameter, macrobend stretch sensors with $\mathrm{N} \geq 5$ were developed to create a practical solution to the pose sensing in soft robot arms. In this arrangement, optical fibres originate from the base and extend to the tip of the robot arm, then loop back to the base to allow placing all required optoelectronics at the base of the arm. This loopback optical fibre system is inherently safe as no electrical current is required at the sensing site, and is suitable for modular implementation in multi-link soft continuum robotic arms. Since this optical fibre arrangement uses a closed optical loop between the emitter and the detector, it is therefore immune to the external lighting conditions. The ability of the macrobend stretch sensors to flexibly adapt their configuration allows preserving the inherent softness and compliance of the robots they are installed on. Other advantages include immunity to electrical noise and magnetic fields. This sensing principle is amenable for integration into to wearable sensing technologies, e.g. pose sensing in the wrist joint of a human hand. The future works will focus on the standardization of the fabrication process of sensors, and further development of the mathematical model to measure the twist of the robotic arm.

\section{Acknowledgments}

The work described in this paper is funded by the Seventh Framework Programme of the European Commission under grant agreement 287728 in the framework of EU project STIFF-FLOP.

\section{Referernces}

Akella, S. and Mason, M.T. (1998) "Posing polygonal objects in the plane by pushing", International Journal of Robotics Research, 17(1), 70-88.

Allsop, T., Dubov, M., Martinez, A., Floreani, F., Khrushchev, I., Webb, D., and Bennion, I. (2005) Long period grating directional bend sensor based on asymmetric index modification of cladding, Electronics Letters, vol. 41, no. 2.

Belardi, W. and. Knight, J.C. (2014) Hollow antiresonant fibres with low bending loss, Opt. Express 22, 10091-10096.

Calisti, M., Giorelli, M., Levy, G., Mazzolai, B., Hochner, B., Laschi, C., Dario, P. (2011) An octopus-bioinspired solution to movement and manipulation for soft robots. Bioinspiration \& biomimetics, 6(3), 036002.

Camarillo, D. B., Milne, C. F., Carlson, C. R., Zinn, M. R., and Salisbury, J. K. (2008) Mechanics modeling of tendon-driven continuum manipulators, IEEE Transactions on Robotics, vol. 24, no. 6, pp. 1262-1273.

Cianchetti, M., Renda, F., Licofonte, A., and Laschi, C. (2012) Sensorization of continuum soft robots for reconstructing their spatial configuration"in Proc. of the IEEE BIOROB, 2012, pp. 634-639.

Cianchetti, M., Ranzani, T., Gerboni, G., De Falco, I., Laschi, C., Menciassi, A. "STIFF-FLOP Surgical Manipulator: mechanical design and experimental characterization of the single module", In proceeding of: IEEE/RSJ International Conference on Intelligent Robots and Systems (IROS), 2013.

Chen, G. (2005) Design, Modeling and Control of a Micro-robotic Tip for Colonoscopy: Onception, Modélisation Et Commande D'un Micro-actionneur Pour la Coloscopie (Doctoral dissertation).

Culha, U., Nurzaman, S., Clemens, F., Iida, F. (2014) SVAS ${ }^{3}$ : Strain Vector Aided Sensorization of Soft Structures, Sensors 2014, 14(7), 12748-12770.

Dobrzynski, M.; Camara, R.P.; Floreano, D (2011). Contactless deflection sensor for soft robots. In Proceedings of theIEEE/RSJ International Conference on Intelligent Robots and Systems, San Francisco, CA, USA, 25-30 September 2011; pp. 19131918.

Feng, J., Zhao, Y., Lin, X.-W., Hu, W., Xu F., Lu, Y.Q. (2011) "A Transflective Nano-Wire Grid Polarizer Based Fibre-Optic Sensor", Sensors, 11(3): 2488-2495.

Flemming, J., Hornes A. (2013) Lissajous-like figures with triangular and square waves, Revista Brasileira de Ensino de F'1sica, v. 35 , n. 3,3702 .

Gutfreund Y, Flash T, Yarom Y, Fiorito G, Segev I, Hochner B. (1996) Organization of octopus arm movements: a model system for studying the control of flexible arms. J Neurosci. 15;16 (22):7297-307.

Hannan, D.M. and Walker, D. I. (2003) "Vision based shape estimation for continuum robots" Proc. Of the 2003 IEEE ICRA (Taipei, Taiwan) pp. $3449-3454$. 
Hoss, R.J., Lacy, E.A. (1993) Fiber Optics, $2^{\text {nd }}$ Edition, ISBN: 0-13-321241-6.

Jay, J. A. (2010) “An overview of macrobending and microbending of optical fibres", White Paper WP1212, Corning, Dec 2010.

Jones, B.A. (2007) Kinematics and implementation of continuum manipulators, $\mathrm{PhD}$ thesis, Clemson University.

Jones, B. A., McMahan, W., and Walker, I. D. (2004) "Design and analysis of a novel pneumatic manipulator," Proceedings of the 3rd IFAC Symposium on Mechatronic Systems, pp. 745-750.

Kim, B., Ha, J., Park, F., Dupont, P. (2014) Optimizing Curvature Sensor Placement for Fast, Accurate Shape Sensing of Continuum Robots. IEEE Int. Conf. Robotics and Automation (ICRA).

Liu, H., Li, J., Song, X., Seneviratne, L. D. and Althoefer, K. (2011) Rolling Indentation Probe for Tissue Abnormality Identification During Minimally Invasive Surgery, IEEE Transactions on Robotics, vol. 27, no. 3, pp. 450-460.

Liu, H., Noonan, D. P. Challacombe, B. J., Dasgupta, P., Seneviratne, L.D., Althoefer, K. (2010) Rolling mechanical imaging for tissue abnormality localization during minimally invasive surgery. IEEE Transactions on Biomedical Engineering, 57(2), 404414.

Liu, C.S., Chou, G.W., Liang, X.M., Reinhall, P.G., and Wang, W.C. (2007) Design of a Multi-layered Optical Bend Loss Sensor for Pressure and Shear Sensing, Proc. of SPIE Smart Structures and Materials \& Nondestructive Evaluation and Health Monitoring, 5768-36.

Lorussi F, Tognetti A, Tesconi M, Pastacaldi P, De Rossi D. (2004) Strain sensing fabric for hand posture and gesture monitoring. Stud Health Technol Inform. 108:266-70.

Malekzadeh, M., Calinon, S., Bruno, D., Caldwell, D.G. (2014) Learning by imitation with the STIFF-FLOP surgical robot: a biomimetic approach inspired by octopus movements, Robotics and Biomimetics 1 (1), 1-15.

Marchese, A. D., Komorowski, K., Onal, C. D., \& Rus, D. (2014). Design and Control of a Soft and Continuously Deformable 2D Robotic Manipulation System. In Proceedings of IEEE International Conference on Robotics and Automation.

Massaro, A., Spano, F., Missori, M., Malvindi, M. A., Cazzato, P., Cingolani, R., and Athanassiou, A. (2014) Flexible nanocomposites with all-optical tactile sensing capability, Rsc adv., vol. 4, iss. 6, p. 2820.

Moro, F.L., Tsagarakis, N.G., and Caldwell, D.G. (2012) On the kinematic Motion Primitives (kMPs) - theory and application, Frontiers in Neurorobotics 6 (10), 1-18.

Murphy, R J., Otake, Y., Taylor, R.S., Armand, M. (2014) Predicting kinematic configuration from string length for a snake-like manipulator not exhibiting constant curvature bending. IROS 2014: 3515-3521.

Nafis, C.A., Jensen, V. Beauregard, L., Anderson, P.T. (2006) "Method for estimating dynamic EM tracking accuracy of Surgical Navigation tools", SPIE Medical Imaging Proceedings.

Noh,Y., Sareh,S., Back, J., Würdemann, H., Ranzani, T., Secco, EL., Faragasso, A., Liu, H., Althoefer, K. (2014) A Three-Axial Body Force Sensor for Flexible Manipulators, IEEE International Conference on Robotics \& Automation (ICRA), 6388 6393.

Noh,Y., Sareh,S., Würdemann, H., Liu, H., Housden, J., Rhode, K., Althoefer, K. (2015) “A three-axial fibre-optic body force sensor for flexible manipulators", IEEE Sensors Journal, Sensors-12865-2015.R1 (accepted paper).

O'Brien, B., Gisby, T., Anderson, I. (2014) Stretch sensors for human body motion, Proc. SPIE 9056, Electroactive Polymer Actuators and Devices (EAPAD) 2014, 905618.

Omega Engineering (1998) Transactions in Measurement and Control, Volume 3, Force-related measurements, Pitman Publishing and Omega Press, New York, pp 62-69.

Patel, S., Park, H., Bonato, P., Chan, L., Rodgers, M. (2012) "A review of wearable sensors and systems with application in rehabilitation," J NeuroengRehabil, 9:21.

Patrick, H., Chang C., and Vohra, S. (1998) Long period fibre gratings for structural bend sensing, Electronics Letters, vol. 34, no. 18, pp. 1773-1775.

Paul, C. (2006) Morphological computation: A basis for the analysis of morphology and control requirements, Robotics and Autonomous Systems 54 (2006) 619-630.

Pfeifer, R., and Iida, F. (2009) Morphological computation: connecting body, brain, and environment, Lecture Notes in Computer Science Volume 5436, 2009, pp 66-83.

Polygerinos, P., Zbyszewski, D., Schaeffter, T., Razavi, R., Seneviratne, L., and Althoefer, K. (2010) “MRI-compatible fiber-optic force sensors for catheterization procedures," IEEE Sensors J., vol. 10, no. 10, pp. 1598-1608.

Ramanujan, Srinivasa, (1914). "Modular Equations and Approximations to $\pi$ ". Quart. J. Pure App. Math. 45: 350-372.

Ranzani, T., Gerboni, G., Cianchetti, M., Menciassi, A. (2015a) A bioinspired soft manipulator for minimally invasive surgery, Bioinspir. Biomim. 10035008.

Ranzani, T., Cianchetti, M., Gerboni, G., De Falco, I., Menciassi, A. (2015b) “A Soft Modular Manipulator for Minimally Invasive Surgery: Design and Characterization of a Single Module”, IEEE Transactions on Robotics (under review paper).

Rieffel J.A., Valero-Cuevas, F.J., Lipson, H.(2010) Morphological communication: exploiting coupled dynamics in a complex mechanical structure to achieve locomotion. J R Soc Interface. 7, 613-21.

Roesthuis, R. J., Janssen, S., Misra, S. (2013) "On using an array of fibre Bragg grating sensors for closed-loop control of flexible minimally invasive surgical instruments," IEEE/RSJ International Conference on in Intelligent Robots and Systems (IROS), pp. 2545-2551.

Ryu, S., Dupont, P. E. (2014) "FBG-based Shape Sensing Tubes for Continuum Robots", IEEE Int. Conf. Robotics and Automation, pp. 3531-3537. 
Sareh,S, Jiang, A., Faragasso, A., Noh, Y., Nanayakkara, T., Dasgupta, P., Seneviratne, L.D., Wurdemann, H.A, Althoefer, K. (2014) Bio-inspired tactile sensor sleeve for surgical soft manipulators, IEEE International Conference on Robotics \& Automation (ICRA), 1454-1459.

Sareh, S., Rossiter, J.M., Conn, A.T., Drescher, K., Goldstein' R.E. (2013) Swimming like algae: biomimetic soft artificial cilia, Journal of the Royal Society Interface 20120666.

Sareh, S., Noh, Y., Ranzani, T., Wudermann, H., Liu, H., Althoefer, K. (2015a) Modular fiber-optic shape sensor for articulated surgical instruments, Hamyln Symposium on Medical Robotics, London, UK.

Sareh, S., Noh, Y., Ranzani, T., Liu, H., Althoefer, K., (2015b) A 7.5mm Steiner chain fiber-optic system for multi-segment flex sensing, IEEE/RSJ International Conference on Intelligent Robots and Systems (IROS).

Saunders, F., Golden, E., White, R. D., Rife, J. (2011) Experimental verification of soft-robot gaits evolved using a lumped dynamic model. Robotica 29(6): 823-830.

Searle, T.C., Althoefer, K., Seneviratne, L.D., Liu, H. (2013) An optical curvature sensor for flexible manipulators. ICRA 2013: $4415-4420$.

Silva, A.S, Catarino, A., Correia, M.V., Frazao, O. (2013) Design and characterization of a wearable macrobending fibre optic sensor for human joint angle determination, Opt. Eng. 52(12), 126106.

Stilli, A., Wurdemann, H.A., Althoefer, K. (2014) Shrinkable, stiffness-controllable soft manipulator based on a bio-inspired antagonistic actuation principle, 2014 IEEE/RSJ International Conference on Intelligent Robots and Systems (IROS), 24762481.

SH1001-1.0 Super Eska ${ }^{\mathrm{TM}}$ Polyethylene jacketed optical fiber cord (http://i-fiberoptics.com/pdf/sh1001-1.0.pdf).

Trivedi D, Rahn C, Kier W, Walker I. (2008) Soft robotics: biological inspiration, state of the art, and future research. Appl. Bionics. Biomech. 5, $99-117$.

Vibhute, V.S., and Kshirsaga, A. (2012) Identification of Hysteresis and Relaxation Parameters in Stretch Sensor, 10.1214/IJAEEE/25.

Webster III, R. J., Kim, J. S., Cowan, N. J., Chirikjian, G. S. and Okamura, A. M. (2006) "Nonholonomic modeling of needle steering" The International Journal of Robotics Research vol. 25, pp. 509-525.

William S. Rone, Pinhas Ben-Tzvi (2013) Multi-segment continuum robot shape estimation using passive cable displacement. ROSE 2013: 37-42.

Wang, W. C., Ledoux, W., Sangeorzan, B., Reinhall, P. (2005) A shear and plantar pressure sensor based on fibre-optic bend loss," Journal of Rehabilitation Research and Development, 42(3), 315-326.

Wang,P., Semenova, Y., Wu, Q., Farrell, G. (2011) A fibre-optic voltage sensor based on macrobending structure, Optics \& Laser Technology 43 (2011) 922-925.

Webster III, R. J. and Jones, B. A. (2010) Design and Kinematic Modeling of Constant Curvature Continuum Robots: A Review," International Journal of Robotics Research, vol. 29, no. 13, pp. 1661-1683.

Willis, K., Brockmeyer, E., Poupyrev, I., and Hudson, S. (2012) Printed Optics: 3D Printing of Embedded Optical Elements for Interactive Devices, In Proc. of UIST'12, 589-598.

Xu, R., Asadian, A., Naidu, A.S., and Patel, R.V. (2013) Position Control of Concentric-Tube Continuum Robots using a. Modified Jacobian-Based Approach, International Conference on Robotics and Automation (ICRA), Karlsruhe, Germany.

Yang, Y.J., Cheng, M.Y., Chang, W.Y., Tsao, L.C., Yang, S.A., Shih, W.P., Chang, F.Y., Chang, S.H., Fan, K.C. (2008) An integrated flexible temperature and tactile sensing array using PI-copper films. Sens. Actuators A Phys., 143-153.

Yekutieli Y, Sagiv-Zohar R, Aharonov R, Engel Y, Hochner B, Flash T. (2005) Dynamic model of the octopus arm. I. Biomechanics of the octopus reaching movement. J Neurophysiol. 94(2):1443-58.

Zawawi M. A., O'Keeffe S., Lewis E., (2013) "Intensity-modulated fibre optic sensor for health monitoring applications: a comparative review", Sensor Review, Vol. 33 Iss: 1, pp.57.

Zelman I, Titon M, Yekutieli Y, Hanassy S, Hochner B, Flash T. (2013) Kinematic decomposition and classification of octopus arm movements, Front Comput Neurosci, 7(60).

Zhang, X., Max, J.J., Jiang, X., Yu, L., Kassi, H. (2007) "Experimental investigation on optical spectral deformation of embedded FBG sensors", Proc. SPIE 6478, Photonics Packaging, Integration, and Interconnects VII, 647808. 